\title{
Vascular Anatomy of the Optic Disc: A Cross Sectional Study in a Sri Lankan Sample
}

\author{
Ruwanpathirana $\mathrm{PS}^{1}{ }^{*}$, Sanjayan $\mathrm{A}^{1}$, Dissanayake $\mathrm{MM}^{1}$ \\ ${ }^{\prime}$ Department of Anatomy, Faculty of Medicine, University of Colombo. \\ *pramithrp@gmail.com
}

The optic nerve head receives the blood supply from the central retinal vessels and posterior ciliary vessels. There is a watershed between the two predisposing to ischaemia.

Angiogenesis on the optic disc (OD) is seen in proliferative diabetic retinopathy. Despite the clinical significance, the vascular patterns on the OD have not been established. Fundoscopic images $(n=150)$ of normal individuals, after excluding images with pathologies by an ophthalmologist were evaluated for the vascular anatomy of the OD. Hundred and four images from 54 people were analysed of which $67.3 \%(n=70)$ were of females. The majority $(92.3 \%, \mathrm{n}=96)$ received the main arterial supply through one artery while the rest had two arteries entering. The number of veins exiting were also either one $(84.6 \%, n=88)$ or two. Majority of the eyes had the arteries $(74.0 \%, \mathrm{n}=77)$ and veins $(90.4 \%, \mathrm{n}=94)$ centrally in the optic cup while the rest had them at the cup, disc margin. Only three $(2.9 \%)$ eyes had pre-papillary looping. The arteries arborized leading to 0-6 arteries $($ mode $=4,31.7 \%, \mathrm{n}=33)$ and 0 3 (mode $=0,51.9 \%, n=54)$ arterial junctions to cross the OD margin. Zero to six veins ( mode $=4,36.5 \%, n=38$ ) crossed the margin while 0-3 had a junction (mode $=0,54.8 \%, \mathrm{n}=57$ ). Majority had 4-7(83.7\%, $n=87)$ minor vessels at $\mathrm{OD}$ (range $=3-10)$. The mode and the median were $5(26.9 \%, \mathrm{n}=28)$ and $6(21.2 \%, \mathrm{n}=22)$ respectively. Establishing normal vascular anatomy of the OD contributes to developing digital technology to identify retinal vascular pathologies early. The association between the vascular variations and pathologies should be sought. 